\title{
Lateral stability control of distributed driven electric vehicle based on sliding mode control
}

\author{
Xian $\operatorname{Li}^{1 *}$ \\ ${ }^{1}$ College of Transportation, Shandong University of Science and Technology, Qingdao, Shandong, 266590, China
}

\begin{abstract}
Aiming at the lateral stability control problem of distributed driven electric vehicles under high speed steering condition, a hierarchical control algorithm of direct yaw moment is designed. The upper control takes the 2-DOF vehicle model as the reference model and uses the sliding mode control to obtain the required yaw moment by tracking the desired yaw velocity and the desired vehicle side-slip angle. The lower control optimizes the distribution of four wheel torque with the minimum tire utilization rate. Finally, Carsim/Simulink was used for model building and co-simulation, and the control effect of PID algorithm was compared. The results show the hierarchical control algorithm achieves the expected goal of improving vehicle lateral stability.
\end{abstract}

\section{Introduction}

When the vehicle is in the high speed steering condition, the lateral force on the tire reaches saturation leading to insufficient and excessive steering of the vehicle. The vehicle is prone to sideslip and swing, which will lead to traffic accidents [1-2]. The stability of the vehicle has become one of the key issues in the field of vehicle technology research.

At present, the stability control of distributed driven electric vehicles generally adopts the hierarchical control structure[3]. The upper motion tracking system calculates the required generalized control force and plans the expected motion state of the vehicle according to the input signal of the driver, the feedback of the vehicle's current state, the ideal state of the reference model and other information. Motion tracking control methods mainly include PID control, fuzzy control, model predictive control (MPC), feedforward and feedback control and sliding mode control (SMC). Each method has its advantages and disadvantages. PID controller is commonly used in engineering, which is convenient. Kim[4] et al. calculated vehicle direct yawing moment by PI control theory, and distributed four-wheel torque by differential drive/brake torque coordination control strategy, but the control strategy is only applicable to lowspeed working conditions. Song[5] designed a PID yaw moment controller based on a linear reference model for a four-wheel independently driven racing car. However, the dynamics of distributed electric vehicles has typical nonlinear characteristics, especially in the process of highspeed driving. Fuzzy control is a control method based on experience. In paper[6-7], the additional yaw moment is calculated by using fuzzy algorithm based on deviation value. However, it is difficult to establish control rules in practical application. Sliding mode control has strong robustness for nonlinear and uncertain parameters system.

In this paper, a hierarchical control algorithm of direct yaw moment is proposed for the distributed driven electric vehicles. The upper control is based on sliding mode control for direct yaw moment controller to ensure the system has strong robustness to external interference. The optimal torque distribution strategy was developed by considering the motor constraint and road adhesion limit in the lower conrtol. Finally, simulation analysis is carried out to verify that the direct yaw moment control strategy proposed in this study can effectively improve the vehicle handling stability and driving safety compared with the traditional PID control method.

\section{Hierarchical Control System Structure}

This paper takes the four-wheel hub motor independently driven, front-wheel steer-by-wire electric vehicle as the research object, as shown in Figure 1. Vehicle state information is sent to ECU via CAN bus, sucn as the front wheel angle, electronic throttle, four wheel motor torque and wheel speed, then the ECU calculates four wheel torques and sends them to four hub motor controllers for motor control. 


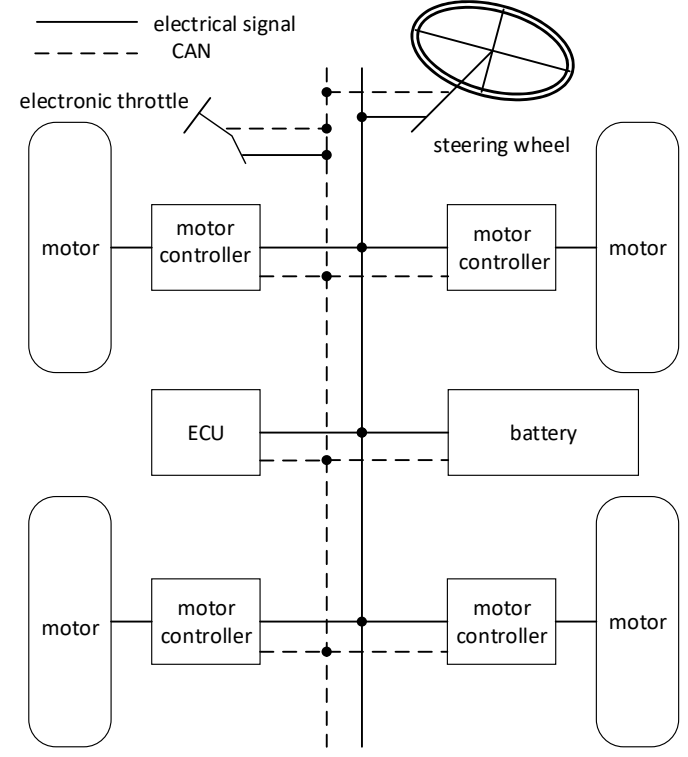

Figure 1. Research object

The institute for the stability of the proposed hierarchical control structure is shown in Figure 2. The upper controller according to the vehicle model output state of vehicle parameters, such as speed $V$, steering angle $\delta$, yaw velocity $\gamma$, vehicle side-slip angle $\beta$, etc, The control target, namely the ideal yaw angle $\gamma_{d}$ and the ideal vehicle side-slip angle $\beta_{d}$, is obtained by using the vehicle 2-DOF dynamic model. Based on the sliding mode control, the additional yaw moment $\triangle M$ required to maintain the vehicle's stable running is calculated and then output to the lower controller. In the lower controller, the minimum tire utilization is the control target, the motor torque, road surface adhesion limit, the longitudinal force and yaw moment needs are the constraint. The controller attachs the upper decision out of yaw moment to optimize allocation, and then gets four rotary torque $\left(T_{f r} T_{f l} T_{r l} T_{r r}\right)$, inputs the vehicle model, achieves the goal of vehicle stability control.

\section{Upper Motion Tracking Controller}

\subsection{Reference Model}

The linear 2-DOF vehicle model can well reflect the basic characteristics of control stability. This paper designs the controller based on the linear two-degree-of-freedom model. The linear 2-DOF vehicle model includes lateral motion and yaw motion. The corresponding motion differential equation (1) is as follows[8]. $k_{f}$ and $k_{r}$ are respectively the lateral stiffness of front and rear wheels, $m$ is the mass of the car, $I_{\mathrm{z}}$ is moment of inertia, $a$ and $b$ are the distances from the front and rear axes to the center of mass, $u$ is the longitudinal velocity.

$$
\left\{\begin{array}{l}
\dot{\beta}=\frac{k_{f}+k_{r}}{m u} \beta+\left(\frac{a k_{f}-b k_{r}}{m u^{2}}-1\right) \gamma-\frac{k_{f}}{m u} \delta \\
\dot{\gamma}=\frac{a k_{f}+b k_{r}}{I_{z}} \beta+\frac{a k_{f}-b k_{r}}{I_{z} u} \gamma-\frac{a k_{f}}{I_{z}} \delta
\end{array}\right.
$$

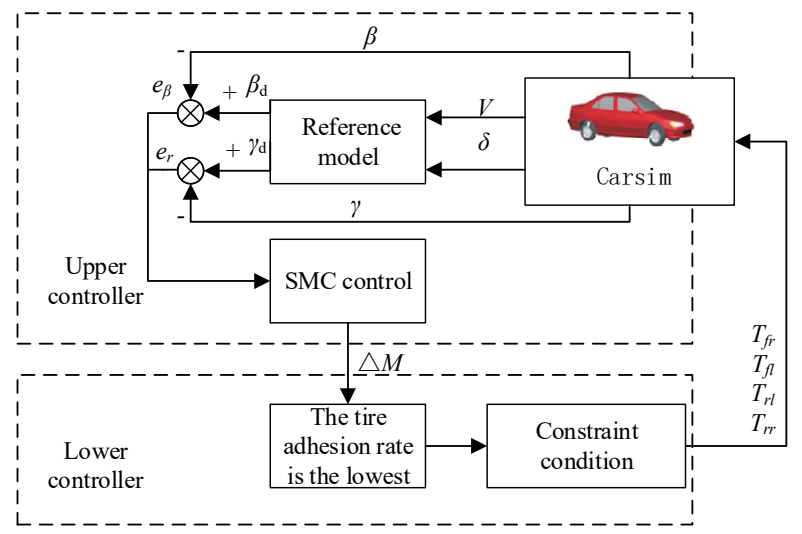

Figure 2. Hierarchical control structure

When the vehicle is turning steady-state, $\dot{v}=0$ and $\dot{\gamma}=0$ can be substituted into equation (1), the desired yaw velocity and the desired vehicle side-slip angle can be deduced.

When the vehicle is running stably, the yaw velocity is:

$$
\gamma=\frac{u / L}{1+k u^{2}} \delta
$$

When the vehicle is running stably, the vehicle sideslip angle is:

$$
\beta=\frac{b+m a u^{2} /\left(k_{r} L\right)}{\left(1+k u^{2}\right) L} \delta
$$

$k$ is a stability factor, $L$ is wheel base.

Considering the adhesion condition of the road surface, $\gamma$ and $\beta$ are limited:

$$
\left|\gamma_{\max }\right|=\left|0.85 \frac{\mu \mathrm{g}}{u}\right|
$$

$\mathrm{g}$ is the gravitational acceleration, $\mu$ is coefficient of road adhesion.

The ideal value of yaw velocity is:

$$
\gamma_{d}=\min \left(|\gamma|,\left|\gamma_{\max }\right|\right) \cdot \operatorname{sgn}\left(\gamma_{d}\right)
$$

Similarly

$$
\beta_{\max }=\tan ^{-1}(0.02 \mu g)
$$

The desired vehicle side-slip angle is:

$$
\beta_{d}=\min \left(|\beta|,\left|\beta_{\max }\right|\right) \cdot \operatorname{sgn}\left(\beta_{d}\right)
$$

\subsection{The Design of Sliding Mode Controller}

By introducing the required yaw moment into equation (1), the following equation (8) can be obtained: 


$$
\left\{\begin{array}{l}
m(\dot{v}+u \gamma)=\left(k_{f}+k_{r}\right) \frac{v}{u}+\frac{1}{u}\left(a k_{f}-b k_{r}\right) \gamma-k_{f} \delta \\
I_{z} \dot{\gamma}=\left(a k_{f}-b k_{r}\right) \frac{v}{u}+\frac{1}{u}\left(a k_{f}+b k_{r}\right) \gamma-a k_{f} \delta+\Delta M
\end{array}\right.
$$

The state space of the equation $(8)$ can be translated into:

$$
\dot{x}=A x+B_{1} \delta+B U
$$

The relevant parameters are:

$$
\begin{aligned}
& x=\left[\begin{array}{ll}
\beta & \gamma
\end{array}\right]^{T} \\
& B=\left[\begin{array}{ll}
0 & \frac{1}{I_{z}}
\end{array}\right]^{T} \\
& B_{1}=\left[\begin{array}{cc}
\frac{2 k_{f}}{m u} & \frac{2 a k_{f}}{I_{z}}
\end{array}\right]^{T} \\
& A=\left[\begin{array}{cc}
-\frac{2\left(k_{f}+k_{r}\right)}{m u} & -1-\frac{2\left(a k_{f}-b k_{r}\right)}{m u^{2}} \\
-\frac{2\left(a k_{f}-b k_{r}\right)}{I_{z}} & -\frac{2\left(a^{2} k_{f}+b^{2} k_{r}\right)}{I_{z} u}
\end{array}\right]^{T}
\end{aligned}
$$

$U=\Delta M$

The sliding mode control theory is used to make the vehicle control variable change with the ideal value of the reference model and then the additional yaw moment is obtained.

The combined deviation of vehicle yaw velocity and vehicle side-slip angle:

$$
e=\left(\gamma_{d}-\gamma\right)+\xi\left(\beta_{d}-\beta\right)
$$

$\xi$ is the weighted coefficient reflecting the control proportion of the vehicle side-slip angle in the control system

The design integral sliding mode surface (sliding mode switching function) is:

$$
s=e+c \int_{0}^{t} e d t
$$

$c$ is a constant which is greater than zero, and $t$ is time. Equation (10) is substituted into equation (11):

$$
s=\left(\gamma_{d}-\gamma\right)+\xi\left(\beta_{d}-\beta\right)+c \int_{0}^{t}\left[\left(\gamma_{d}-\gamma\right)+\xi\left(\beta_{d}-\beta\right)\right] d t
$$

The first derivative of the sliding mode switching function is obtained as follows:

$$
\dot{s}=\left(\dot{\gamma}_{d}-\dot{\gamma}\right)+\xi\left(\dot{\beta}_{d}-\dot{\beta}\right)+c\left[\left(\gamma_{d}-\gamma\right)+\xi\left(\beta_{d}-\beta\right)\right]
$$

According to equation (8), it can be obtained:

$$
\dot{\gamma}=\frac{1}{I_{z}}\left(\left(a k_{f}-b k_{r}\right) \frac{v}{u}+\frac{1}{u}\left(a k_{f}+b k_{r}\right) r-a k_{f} \delta+\Delta M\right)
$$

Equation (14) is substituted into equation (13), when the first derivative of the sliding mode switching function is 0 , the equivalent controller is:

$$
u_{e q}=I_{z}\left[\dot{\gamma}_{d}+\xi\left(\dot{\beta}_{d}-\dot{\beta}\right)+c\left(\left(\gamma_{d}-\gamma\right)+\xi\left(\beta_{d}-\beta\right)\right)\right]-a F_{y f} \cos \delta+b F_{y r}
$$

$F_{y f}$ and $F_{y r}$ are the lateral force of front wheel and rear wheel respectively.

To ensure that the sliding mode condition is valid, the system switching controller is designed as follows:

$$
u_{s w}=-I_{z} K \operatorname{sgn}(s)
$$

The required additional yaw moment is:

$$
\Delta M=u_{e q}+u_{s w}
$$

It is obvious that the stability of sliding mode variable structure control is valid.

\section{Lower Controller of Torque Distribution}

The upper controller obtains the vehicle yaw moment, and the lower controller adopts the moment distribution method based on the minimum sum of tire adhesion utilization to make the vehicle in a good adhesion state and realizes the reasonable distribution of additional yaw moment. The design goal is to minimize the sum of the ground adhesion utilization of the four tires:

$$
\min J=\sum_{i=1}^{4} \frac{F_{x i}^{2}}{\left(\mu F_{z i}\right)^{2}}=\frac{F_{x f l}{ }^{2}}{\left(\mu F_{z f l}\right)^{2}}+\frac{F_{x f r}{ }^{2}}{\left(\mu F_{z f r}\right)^{2}}+\frac{F_{x r l}{ }^{2}}{\left(\mu F_{z r l}\right)^{2}}+\frac{F_{x r r}{ }^{2}}{\left(\mu F_{z r r}\right)^{2}}
$$

\section{Simulation and result analysis}

In this paper, simulink simulation software and carsim vehicle dynamics software are used to establish the cosimulation model to verify the control algorithm. The effectiveness of the proposed control method is verified through the design of double-shift driving condition. The stability and path tracking capability of the vehicle can be better verified under the double-shift driving condition at high speed.

Figure 3 shows the comparison of the vehicle path tracking effect under PID control and sliding mode control (SMC). Compared with the ideal double-line path, the vehicle path tracking error under PID control is relatively large, the maximum error is about $0.4 \mathrm{~m}$. The maximum error of path tracking based on SMC is about $0.1 \mathrm{~m}$. It can be seen that in the path tracking effect, the effect of SMC is better.

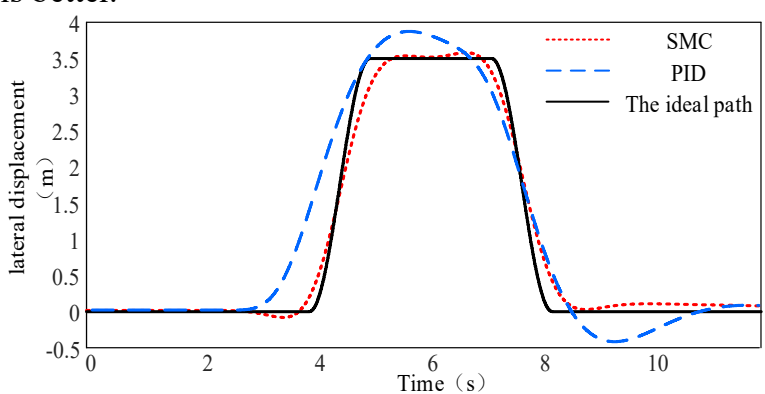

Figure 3. The effect of path tracking

Figure. 4 shows the comparison of yaw velocity under the two control methods. Compared with the desired value of yaw velocity, the peak value of vehicle yaw velocity under PID control is about $7 \mathrm{deg} / \mathrm{s}$, which is too small compared with the ideal yaw velocity. The peak value of vehicle yaw velocity under SMC is around 13 degrees, can better track the desired yaw velocity. 


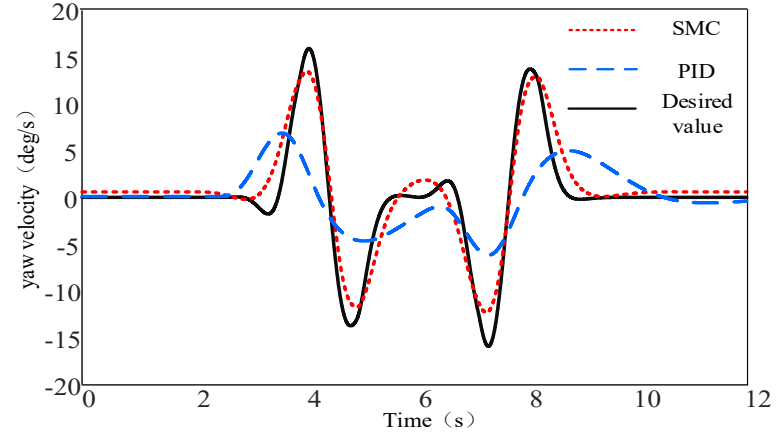

Figure 4. Yaw velocity comparison

Figure 5 shows the comparison of vehicle side-slip angle under the two control strategies. The desired vehicle side-slip angle is 0 , so the smaller vehicle side-slip angle can have the better the vehicle stability. It can be seen from the figure 5 that the peak value of vehicle side-slip angle under SMC is about $0.7 \mathrm{deg}$, and the peak value of vehicle side-slip angle under PID control is about $1.2 \mathrm{deg}$. The stability of SMC is better than PID control.

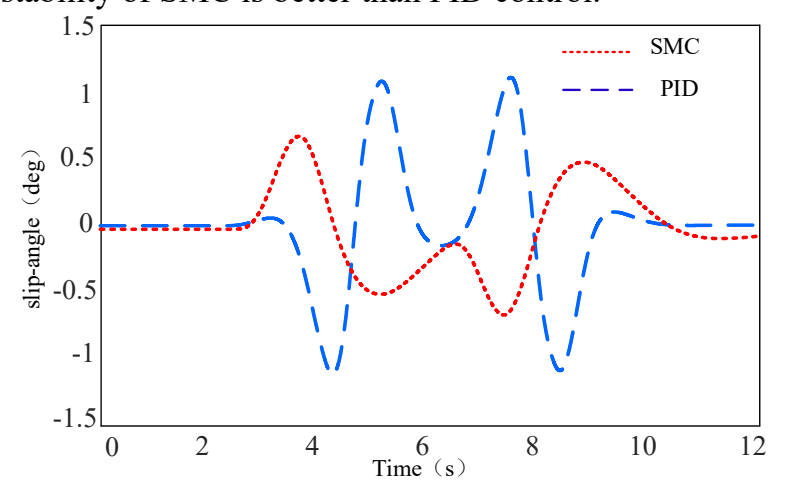

Figure 5. Vehicle side-slip angle

\section{Conclusion}

In this paper, a direct yaw moment hierarchical control algorithm is designed for stability control of distributed driven electric vehicles. The upper control uses sliding mode control to obtain the required yaw moment. The lower controller distributes the four wheel torques optimally with the goal of minimizing tire utilization. The simulation results show that the SMC strategy has better real-time performance and self-adaptability under the condition of high speed steering, and achieves the expected goal of improving the lateral stability of vehicles.

\section{References}

1. Yan Y, Ye G, Zhang Z. Yaw Control of Electric Wheel Vehicle Based on Model Predictive Control, Journal of Wuhan University of Science and Technology, 2018,41(3): 226-231.

2. Alipour H, Sabahi M, Sharifian M B B. Lateral stabilization of a four-wheel independent drive electric vehicle on slippery roads, Mechatronics,2015,30:275-285.

3. Yu Z, Feng Y, Xiong L. Review on Vehicle Dynamics
Control of Distributed Drive Electric Vehicle, Journal of Mechanical Engineering,2013,49(8):105-114.

4. Kim W, Kyongsu Y, Jongseok L. Drive Control Algorithm for An Independent 8 In-wheel Motor Drive Vehicle, Journal of Mechanical Science \& Technology,2011,25(6):1573-1581.

5. Song C, Li G. Stability Control of Four-Wheel Independent Drive FSEC Racing Car,Auto Engineer,2020,(7):31-34.

6. Fan X, Fan B, Ang H W. Fuzzy Control for Vehicle Stability, Artificial Intelligence,Management Science and Electronic Commerce. 2011,9(5):2115-2118.

7. Chen Y, Hedrick J K, Guo K. A Novel Direct Yaw Moment Controller for In-wheel Motor Electric Vehicles, Vehicle System Dynamics,2013,51(6):958942.

8. $\mathrm{Yu}$ Z. Automobile Theory, China Machine Press,2018,Beijing. 